\title{
Insufficienza renale cronica di medio grado ed escrezione urinaria di alluminio e fosforo dopo carico farmacologico acuto
}

\author{
B. Di Iorio ${ }^{1}$, V. Terracciano ${ }^{1}$, A. Bruno ${ }^{2}$, G. Cosentino ${ }^{3}$, D. Papaleo ${ }^{3}$, F. Smilari², \\ V. Bellizzi, C. Altieri, G. Gaudiano \\ ${ }^{1}$ U.O. di Nefrologia e Dialisi, Ospedale di Lauria $(\mathrm{Pz})$ \\ ${ }^{2}$ Laboratorio Analisi Chimiche, Castrovillari (Cs) \\ ${ }^{3}$ Laboratorio di Analisi, Ospedale di Lauria
}

1 problema alluminio $(\mathrm{Al})$ nasce negli anni '70 quando un gruppo di ricercatori di Denver dimostrò la presenza di elevate concentrazioni di Al nella sostanza grigia cerebrale di uremici in dialisi in cui si era manifestato un quadro clinico particolare di encefalopatia progressiva.

Nell'organismo umano, in condizioni fisiologiche, il contenuto totale di $\mathrm{Al}$ non supera i $30 \mathrm{mg}$ e, poiché il rene rappresenta praticamente l'unico organo escretore, nel paziente uremico si verifica un accumulo progressivo che può raggiungere anche i 3 grammi. Tale deposito interessa una vasta gamma di organi: osso, encefalo, midollo osseo, muscoli, articolazioni, apparato gastroenterico, miocardio, polmoni, cute, fegato.

Pur nell'ambito di questa ampia distribuzione, i distretti dove l'accumulo di Al configura quadri anatomoclinici attualmente ben definiti sono:
1. L'apparato scheletrico;

2. Il sistema nervoso centrale;

3. Il sistema emopoietico.

L'apporto medio di Al con gli alimenti e con l'acqua è di circa 24-36 $\mathrm{mg} / \mathrm{die}$ (ma vi sono anche lavori che forniscono stime inferiori, dell'ordine di 3-5 mg). Solo una piccolissima parte della quantità ingerita viene assorbita, circa $15-30 \mathrm{mcg}$. Vari fattori possono influenzare l'assorbimento gastro-enterico di Al: il PTH, la vitamina $\mathrm{D}$, altri oligoelementi quali il fluoro, e metalli quali il ferro, e, infine, diversi costituenti della dieta quali il citrato, il lattato, l'ascorbato, l'acido malico, l'ossalato (1-5). Ma, soprattutto, sono importanti le caratteristiche chimico-fisiche di Al: esso è presente, $\mathrm{a} \mathrm{pH} 4.2$, in forma libera, assorbibile $100-1000$ volte più che a pH 6.2-8.1. Ciò significa che l'assorbimento di $\mathrm{Al}$ è favorito dal $\mathrm{pH}$ acido, fisiologico, del tratto gastroenterico prossimale (1-5). Abbiamo già dimostrato che la riduzione farmacologica dell' acidità gastrica induce una minore escrezione urinaria di $\mathrm{Al}$ in soggetti normali (6-8), ma non in pazienti con insufficienza renale cronica (IRC) (8-10). Se si aumenta l'introduzione orale di $\mathrm{Al}$ con un carico addizionale di 1-3 g/die, anche nei normali il bilancio di Al diventa positivo (11). Già Sàrszegi et al recentemente hanno dimostrato che, in pazienti con moderata o severa IRC, l'assorbimento di $\mathrm{Al}$ è protratto, causandone elevati livelli sierici per un tempo prolungato (12). Perciò, l'assunzione cronica di cibi o, soprattutto, di farmaci contenenti Al può essere alla base di una tossicità da $\mathrm{Al}$, già in una condizione di IRC moderata $(12,13)$. Comunque, nei pazienti con IRC di grado iniziale-medio è stato poco investigato questo aspetto, nonostante l'uso regolare di Idrossido di Alluminio - $\mathrm{Al}(\mathrm{OH})_{3}$, almeno nel passato. 
TABELLA I - DATI BASALI DELLO STUDIO NELLA POPOLAZIONE CONTROLLO E NEI PAZIENTI CON IRC DI MEDIO GRADO

\begin{tabular}{lccc}
\hline & Controlli $(\mathrm{n}=10)$ & IRC $(\mathrm{n}=12)$ & $\mathrm{P}$ \\
\hline $\mathrm{Al}-\mathrm{s}(\mathrm{mcg} / \mathrm{L})$ & $5.5 \pm 2.12$ & $9.5 \pm 0.7$ & 0.0000 \\
\hline $\mathrm{Cl}-\mathrm{Al}$ & $4.1 \pm 1.1$ & $2.05 \pm 1.5$ & 0.0018 \\
$\mathrm{Al}-\mathrm{u} / \mathrm{Cr}-\mathrm{u}$ & $0.412 \pm 0.046$ & $0.046 \pm 0.042$ & 0.0000 \\
$\mathrm{Fe}-\mathrm{Al}(\%)$ & $0.043 \pm 0.059$ & $0.215 \pm 0.264$ & 0.0014 \\
$\mathrm{Al}-\mathrm{u}(\mathrm{mcg} / \mathrm{min})$ & $0.086 \pm 0.036$ & $0.129 \pm 0.05$ & 0.0345 \\
$\mathrm{P}-\mathrm{s}(\mathrm{mg} / \mathrm{dl})$ & $3.35 \pm 0.54$ & $4.46 \pm 1.42$ & 0.0305 \\
$\mathrm{Fe}-\mathrm{P}(\%)$ & $0.14 \pm 0.029$ & $0.523 \pm 0.11$ & 0.0000 \\
$\mathrm{P}-\mathrm{u}(\mathrm{mg} / \mathrm{min})$ & $0.65 \pm 0.18$ & $0.33 \pm 0.14$ & 0.0000 \\
\hline
\end{tabular}

Scopo del lavoro è la valutazione delle variazioni sieriche ed urinarie di $\mathrm{Al}$ indotte da un carico acuto di $\mathrm{Al}(\mathrm{OH})_{3}$ nei pazienti con IRC iniziale.

Abbiamo anche valutato le variazioni sieriche ed urinarie dei fosfati $(\mathrm{P})$, indotte dal ridotto assorbimento intestinale per l'uso del chelante (14).

\section{Materiali e metodi}

Abbiamo studiato un gruppo di 10 volontari sani $(5 \mathrm{M}, 5 \mathrm{~F}$, età media \pm DS: $38.45 \pm 11$ anni, clearance creatinina: $120 \pm 10 \mathrm{ml} / \mathrm{min}$ ) ed un gruppo di 12 pazienti con IRC $(6 \mathrm{M}, 6 \mathrm{~F}$, $42.32 \pm 10.22$ anni, ClCr: $40.4 \pm 8.8$ $\mathrm{ml} / \mathrm{min}$, range: $60-30 \mathrm{ml} / \mathrm{min}$ ). Per quattro settimane consecutive, tutti i soggetti hanno seguito una dieta normocalorica, adeguata all'età e al peso corporeo e bilanciata nella composizione (proteine: $1 \mathrm{~g} / \mathrm{kg} \mathrm{pc} / \mathrm{die}$, carboidrati e lipidi in quota equivalente, rispettivamente, al $67-70 \%$ ed al 30 $35 \%$ del restante contenuto calorico). Non sono stati somministrati farmaci contenenti Al. Dei pazienti con IRC, 5 erano affetti da glomerulopatie, 4 da nefroangiosclerosi e 3 da nefropatie interstiziali. Tutti i pazienti erano, al momento dello studio, senza patologie acute in atto, con peso stabile e con volume urinario senza particolari variazioni nei tre mesi precedenti (dati ricavati dai controlli ambulatoriali). Infine, tutti presentavano una proteinuria inferiore a $1 \mathrm{~g} / 24 \mathrm{~h}$, che costituiva il limite di esclusione dallo studio. Alla fine della quarta settimana, sono stati raccolti campioni di sangue e dell'urina di $24 \mathrm{~h}$. È stato effettuato il dosaggio di protidemia totale, albuminemia e PTH nei due gruppi, che non ha mostrato differenze statistiche significative. Poi, dopo che sia i soggetti controllo (C) che i pazienti (IRC) hanno assunto $2 \mathrm{~g}$ di $\mathrm{Al}(\mathrm{OH})_{3}$ per os a digiuno, sono state effettuate campionature di urina e siero ai tempi: $0,1,2,3,4,8,12,16,24$ ore. Su tutti i prelievi di siero (s) e di urina (u) sono stati dosati: $\mathrm{Al}, \mathrm{Cr}$ e $\mathrm{P}$. La determinazione di $\mathrm{Al}$ è stata effettuata mediante spettrometro elettrotermico ad assorbimento atomico (mod. Spectra 30), presso il Laboratorio di Analisi Chimiche della A.S.L. di Castrovillari (CS). Questo Laboratorio partecipa al programma di controllo di qualità, tenuto, su scala nazionale, dall'Istituto Superiore di Sanità. In tutte le varie fasi della procedura analitica, è stata adottata ogni precauzione atta ad evitare contaminazioni da alluminio estraneo, quali: l'uso di acqua ultrapura, di reagenti ad elevato grado di purezza e di materiale plastico, il risciacquo di tutto il materiale di laboratorio con soluzione di acido nitrico al $10 \%$, lo scarto di reagenti e contenitori sospetti di contaminazione da $\mathrm{Al}$, riduzione al minimo indispensabile di ogni manipolazione dei campioni ed, infine, valutazione in parallelo di "bianchi". Cr e P sono stati misurati mediante autoanalyzer (Hitachi mod. 704, Boehringher Mannheim, Ger- mania).

La valutazione statistica è stata effettuata mediante l'analisi della varianza ed il test $t$ di Student per dati appaiati e non.

\section{Risultati}

La Tabella I mostra i dati basali dello studio nelle due popolazioni: i pazienti con IRC mostrano livelli sierici di $\mathrm{Al}$ significativamente più alti $(9.5 \pm 0.7 \mathrm{mcg} / \mathrm{L})$, una $\mathrm{Cl}-\mathrm{Al}$ significativamente più bassa $(2.01 \pm 1.1$ $\mathrm{ml} / \mathrm{min}$ ), un rapporto urinario $\mathrm{Al} / \mathrm{Cr}$ più basso $(0.046 \pm 0.042)$, un'escrezione frazionata di $\mathrm{Al}$ più alta $(0.129$ \pm 0.05 ), un'escrezione urinaria minutata di Al più alta $(0.129 \pm 0.05$ $\mathrm{mcg} / \mathrm{min}$ ), livelli sierici di $\mathrm{P}$ più alti $(4.46 \pm \mathrm{mg} / \mathrm{dl})$, un'escrezione frazionata di $\mathrm{P}$ più alta $(0.523 \pm 0.11) \mathrm{e}$ un'escrezione urinaria minutata di $\mathrm{P}$ più bassa $(0.33 \pm 0.14 \mathrm{mg} / \mathrm{min})$.

La Figura 1 mostra i livelli sierici di $\mathrm{Al}(\mathrm{mcg} / \mathrm{L})$ dopo l'assunzione di $2 \mathrm{~g}$ di idrossido di Al per os: nei controlli vi è un lento aumento dei livelli sierici fino alla terza ora e poi un lento decremento e un plateau dopo la dodicesima ora $(\mathrm{F}=5.87, \mathrm{p}<0.01)$; invece in IRC vi è la presenza di un doppio picco ematico dopo la prima ora e verso l'ottava ora con un lento decremento che inizia dopo la sedicesima ora $(\mathrm{F}=6.56, \mathrm{p}=0.007)$.

La Figura 2 mostra il rapporto urinario $\mathrm{Al} / \mathrm{Cr}$ : nei controlli vi è un picco nella seconda ora e un rapido ritorno 


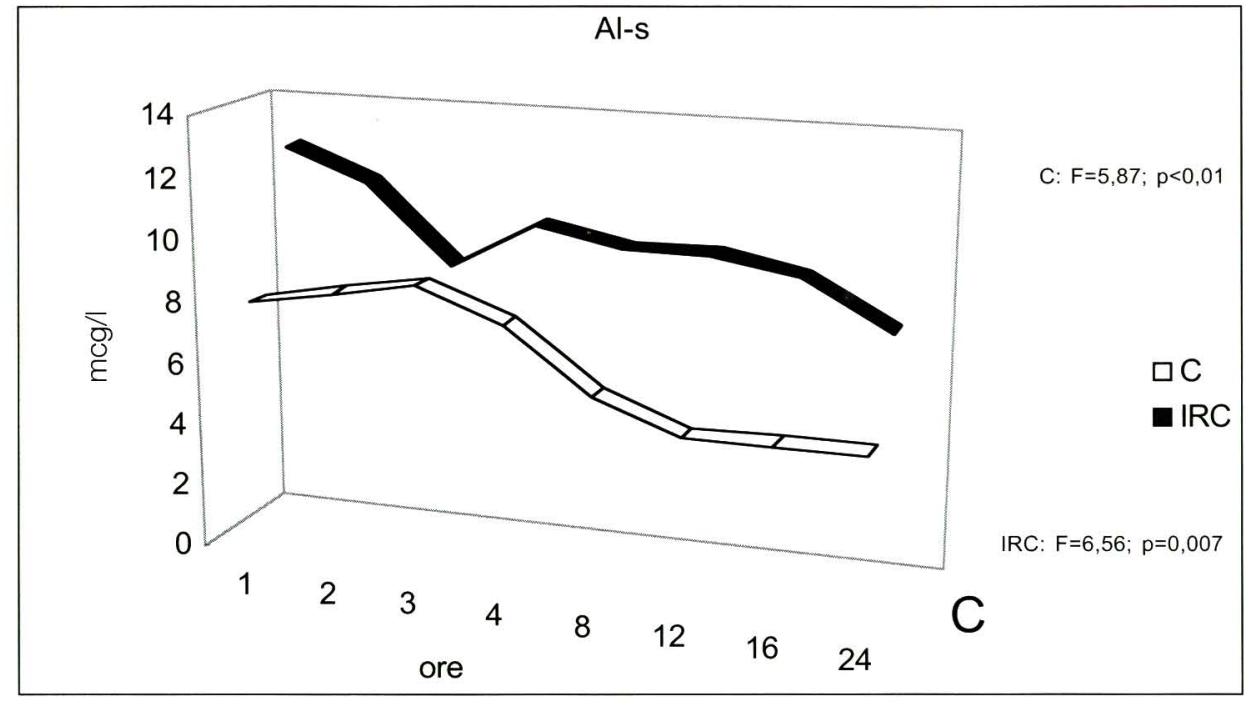

Fig. 1 - Variazione dei livelli sierici di Al (mcg/L) dopo l'assunzione di $2 \mathrm{~g}$ di idrossido di Al per os ai vari tempi dello studio (IRC versus Controlli $p<0.001$ ai tempi 1, 2, 3, 4, 8, 12, 16, 24 ore).

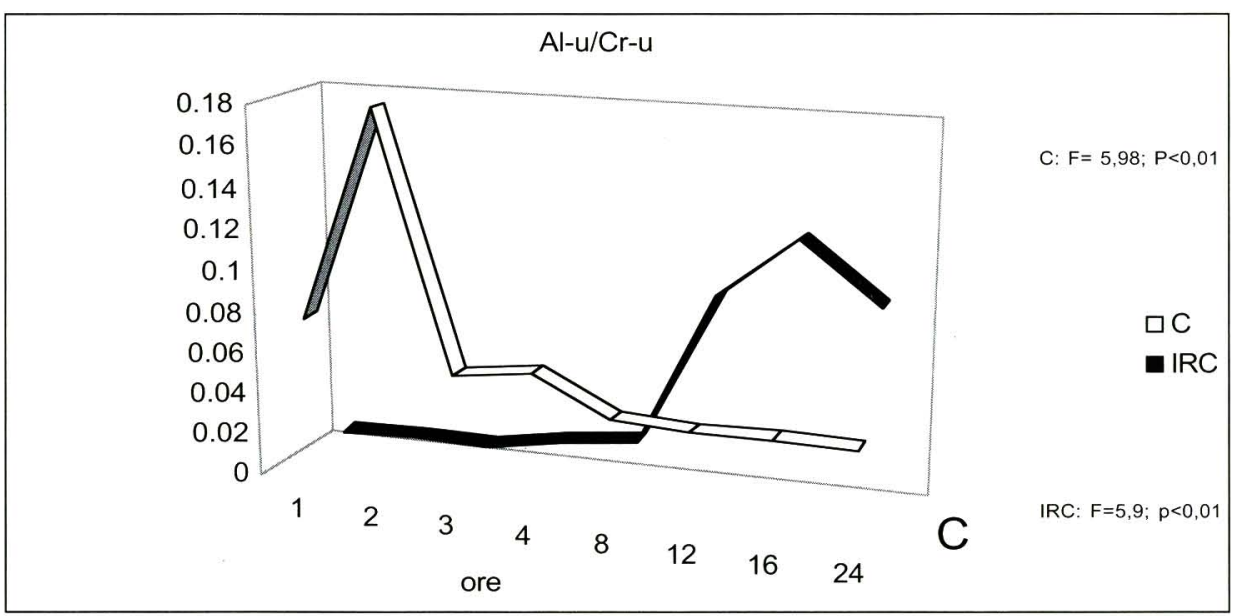

Fig. 2 - Variazione del rapporto urinario Al/Cr dopo l'assunzione di $2 \mathrm{~g}$ di idrossido di Al per os ai vari tempi dello studio (IRC versus Controlli $p<0.001$ ai tempi 1, 2, 3, 4, 8, 12, 16, 24 ore).

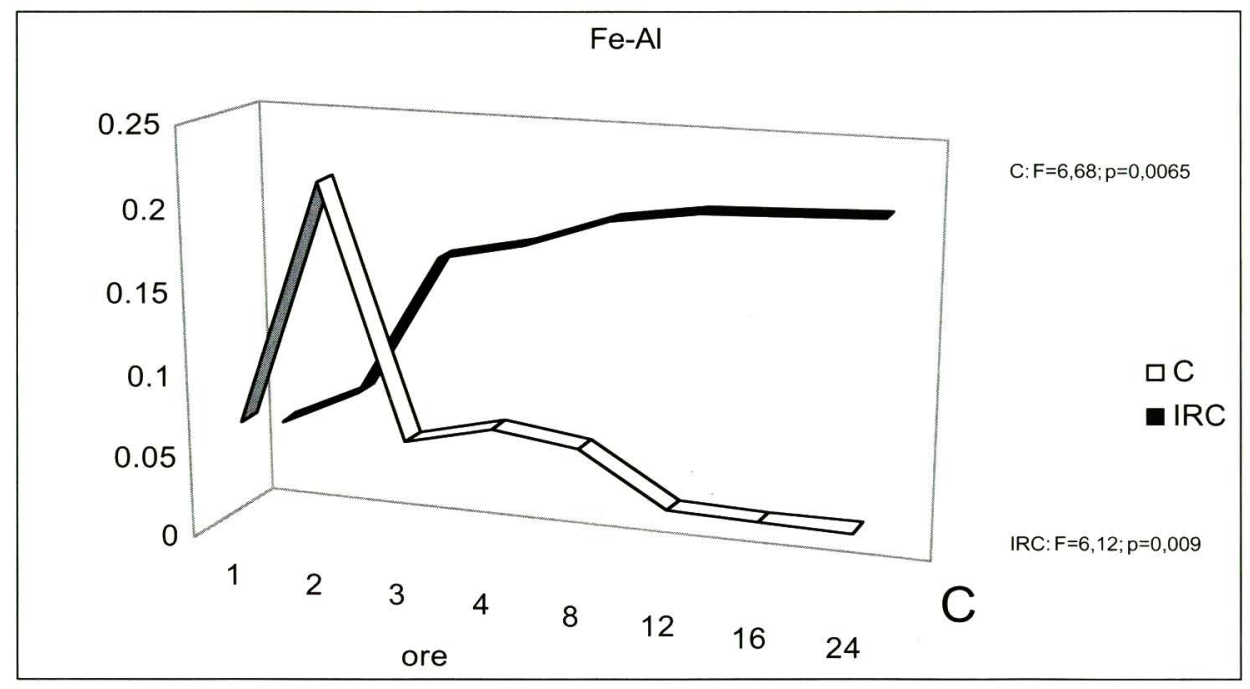

Fig. 3 - Variazione dell'escrezione frazionata di Al dopo l'assunzione di $2 \mathrm{~g}$ di idrossido di Al per os ai vari tempi dello studio (IRC versus Controlli $p<0.001$ ai tempi 1, 2, 3, 4, 8, 12, 16, 24 ore). ai valori basali dalla terza ora $(\mathrm{F}=5.98, \mathrm{p}<0.01)$; invece in IRC vi è un tardo e lento aumento di tale rapporto dopo le prime otto ore di osservazione $(\mathrm{F}=5.9, \mathrm{p}<0.01)$.

La Figura 3 mostra l'escrezione frazionata di $\mathrm{Al}$ : nei controlli vi è un rapido aumento di Fe-Al nella seconda ora e un rapido ritorno ai valori basali dalla terza ora $(\mathrm{F}=6.68, \mathrm{p}=0.0065)$; invece in IRC vi è un tardo e lento aumento di tale rapporto dalla quarta ora con un plateau dopo le prime 12 ore $(\mathrm{F}=6.12, \mathrm{p}=0.009)$.

La Figura 4 mostra la clearance di $\mathrm{Al}$ nei due gruppi di pazienti: nei controlli vi è un rapido picco nelle prime due ore che mostra una riserva funzionale di circa $16 \mathrm{ml} / \mathrm{min}$ (aumento di circa 5 volte) e un ritorno ai valori basali dopo le prime 8 ore, invece in IRC vi è solo un lento continuo aumento della clearance che non raggiunge i $2 \mathrm{ml} / \mathrm{min}$

La Figura 5 mostra i livelli sierici di $\mathrm{P}(\mathrm{mg} / \mathrm{dl})$ dopo l'assunzione di $2 \mathrm{~g}$ di idrossido di Al per os: nei controlli vi è un picco dei livelli sierici durante la terza ora e un successivo rapido decremento senza che, però, tale modificazione avesse una significatività $(\mathrm{F}=0.084, \mathrm{p}=\mathrm{NS})$; invece in IRC vi è una lenta discesa durante tutto il periodo di osservazione $(\mathrm{F}=0.094$, $\mathrm{p}=\mathrm{NS}$ ).

La Figura 6 mostra l'escrezione urinaria minutata di P: nei controlli vi è un picco nella prima ora con un aumento del $60 \%$ dell'escrezione di $\mathrm{P}$ e un rapido ritorno ai valori basali senza significatività statistica $(\mathrm{F}=0.101, \mathrm{p}=\mathrm{NS})$; in IRC il picco nella prima ora è di minore entità $\mathrm{e}$ senza significatività statistica $(\mathrm{F}=0.101 ; \mathrm{p}=\mathrm{NS})$.

\section{Conclusioni}

Gli uremici hanno due possibili importanti fonti di esposizione all'Al: il dialisato e i chelanti del fosforo che contengono idrossido di Al. L'uremia faciliterebbe l'assorbimento di $\mathrm{Al}$ in seguito ad alterazioni gastroenteriche proprie dell'uremia stessa (7-10). Inoltre lo stato di deposito del ferro modulerebbe l'assorbimento di Al, 
soprattutto i livelli di ferritina condizionerebbero in modo inversamente proporzionale l' assorbimento di Al.

La barriera intestinale e l'escrezione renale rappresentano fisiologicamente i due principali organi di controllo della concentrazione di Al nell'organismo. Dal momento che non è disponibile un isomero radioattivo di Al, gli studi di bilancio di entrate ed uscite sono basati su valutazioni indirette mediate dalla determinazione dei livelli plasmatici, dell'escrezione urinaria e delle concentrazioni tissutali (11). L'assorbimento gastroenterico di $\mathrm{Al}$, con tali studi, in soggetti con normofunzione renale, è stimato essere 1'1-3\% di quanto introdotto per via orale. Se si aumenta tale apporto, con un carico addizionale di 1$3 \mathrm{~g} / \mathrm{die}$, anche nei normali il bilancio di Al diventa positivo $(1-3,10,15)$. Nostri studi confermano tali osservazioni e confermano che un carico orale di Al è causa di incremento dei suoi livelli sierici (Al-s) ed urinari (Al-u), sia in controlli normali che in pazienti con IRC $(6-9,16,17)$. Abbiamo dimostrato che un carico cronico di $\mathrm{Al}(\mathrm{OH})_{3}$ (4 g/die per 7 giorni) comporta una riduzione di $\mathrm{Cl}-\mathrm{Al}$ del $61 \%$ ed una riduzione di $\mathrm{Fe}-\mathrm{Al}$ del $77 \%$ nei soggetti con moderata IRC (16). Inoltre, il rapporto urinario $\mathrm{Al} / \mathrm{Cr}$ aumenta del $341 \%$ nelle fasi iniziali e del $398 \%$ nelle fasi avanzate di IRC (27), e l'uso di terapia conservativa con chetoanaloghi normalizza l'escrezione urinaria di $\mathrm{Al}$ che il rapporto urinario $\mathrm{Al} / \mathrm{Cr}$ probabilmente per un minor intake di $\mathrm{Al}(16,17)$.

Sàrszegi et al recentemente hanno dimostrato che, in IRC moderata o severa, l'assorbimento di $\mathrm{Al}$ è protratto, causando alti livelli di Al-s per un tempo prolungato (12). Perciò, il cronico apporto di $\mathrm{Al}$, con cibi o con farmaci che lo contengono, può causare un quadro di tossicità anche nell'IRC moderata $(12,13)$. La valutazione di questi Autori è confortata solo dai dati di Al sierico. Convinti che anche una condizione di IRC iniziale può determinare un accumulo di $\mathrm{Al}$, in caso di sovra-esposizione (6-9, 16-18), abbiamo ripetuto lo stesso schema sperimentale di Sàrszegi (12), valutando, però, anche

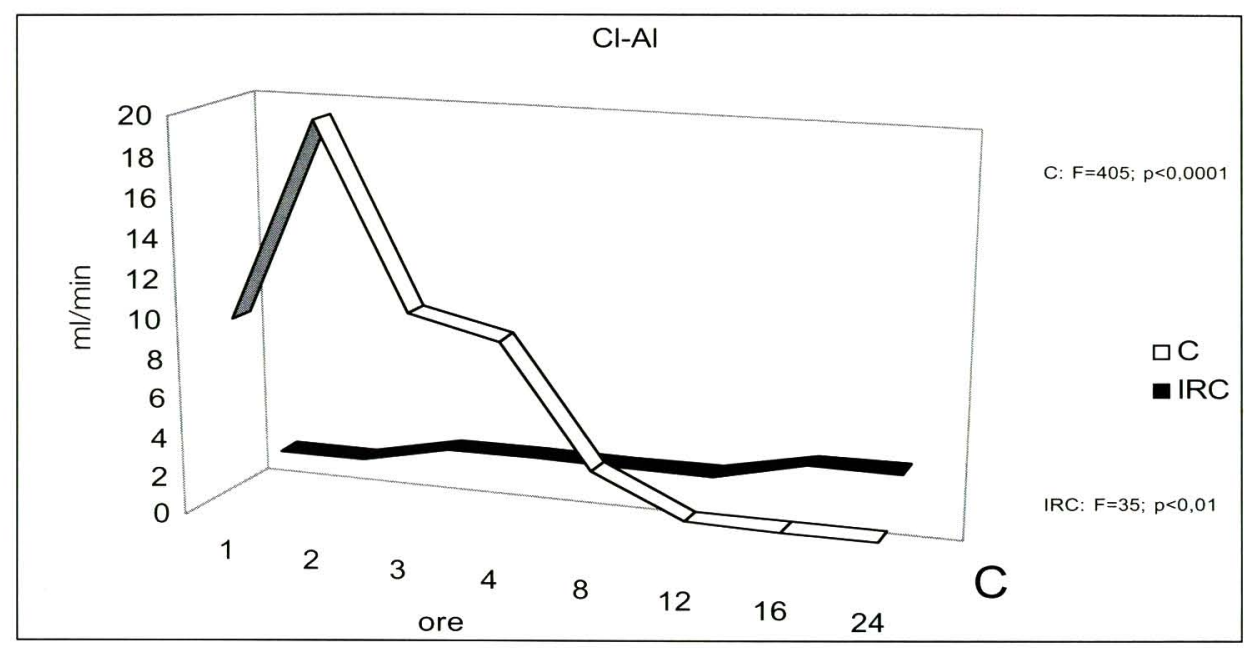

Fig. 4 - Variazione della clearance di Al dopo l'assunzione di $2 \mathrm{~g}$ di idrossido di Al per os ai vari tempi dello studio (IRC versus Controlli $p<0.001$ ai tempi 1, 2, 3, 4, 8, 16, 24 ore).

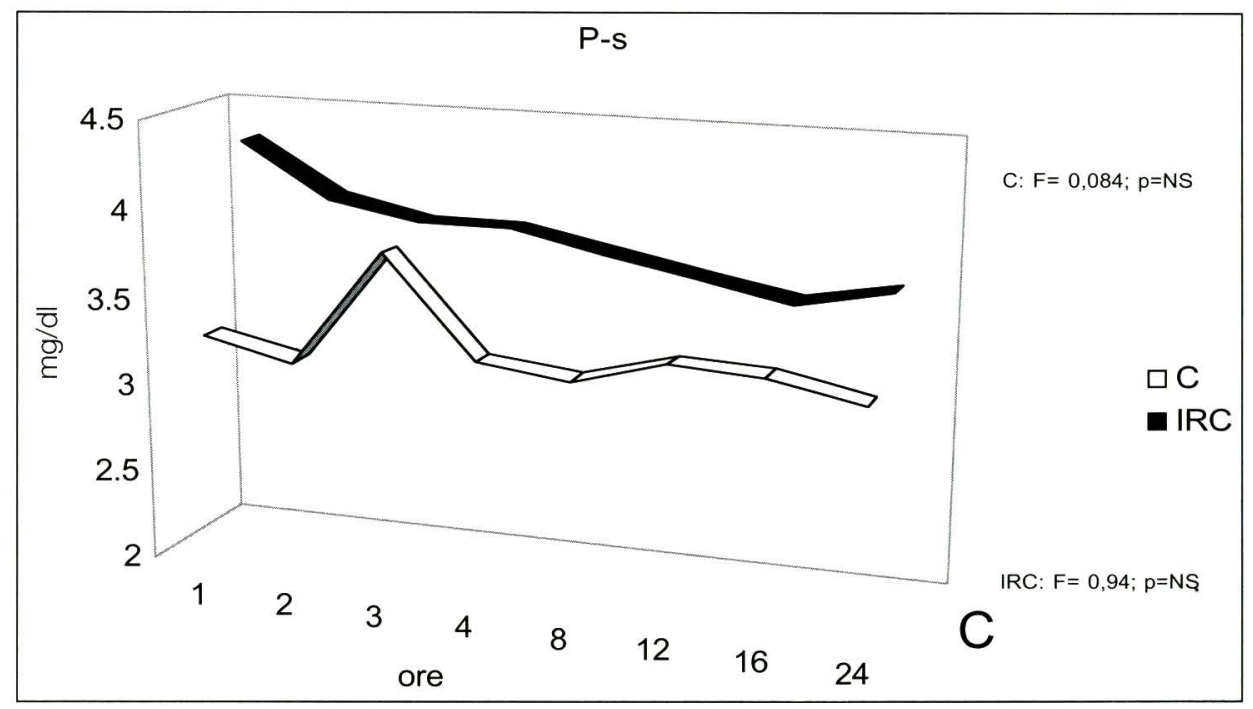

Fig. 5 - Variazione dei livelli sierici di $P$ ( $\mathrm{mg} / \mathrm{dl}$ ) dopo l'assunzione di $2 \mathrm{~g}$ di idrossido di $\mathrm{Al}$ per os (IRC versus Controlli $p=N S$ ai tempi 1,2, 3, 4, 8, 12,16, 24 ore).

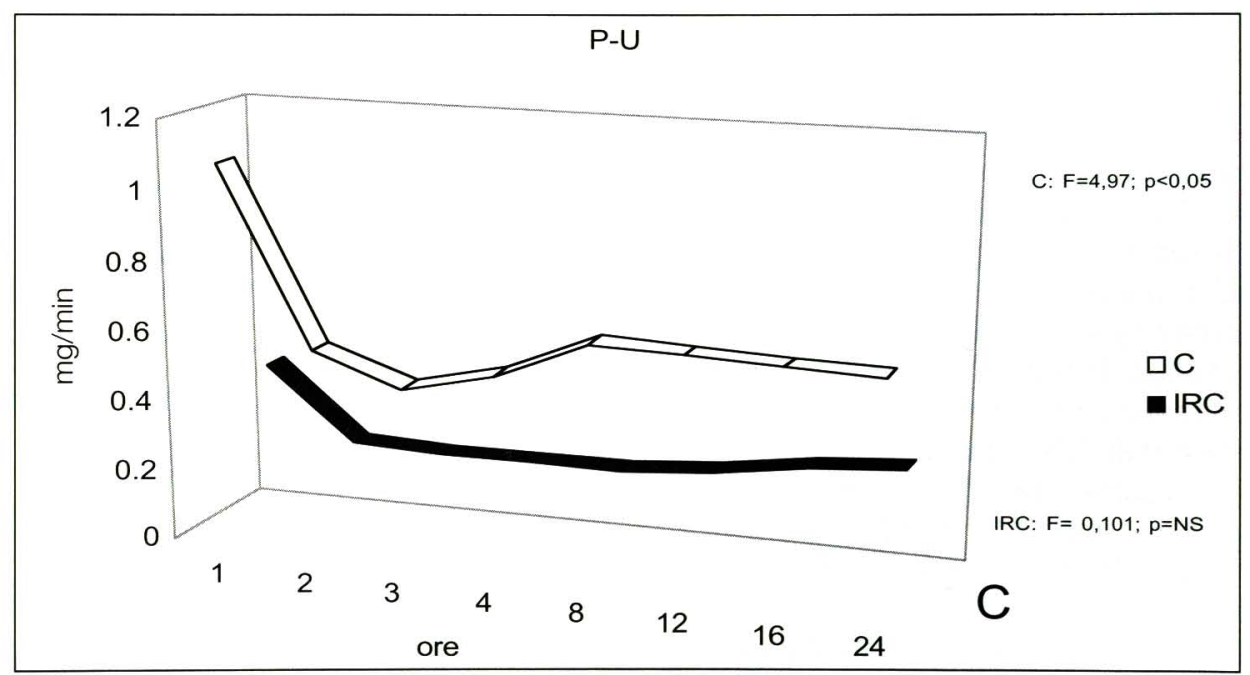

Fig. 6 - Variazione dell'escrezione urinaria minutata di P dopo l'assunzione di $2 \mathrm{~g}$ di idrossido di Al per os ai vari tempi dello studio (IRC versus Controlli $p<0.001$ ai tempi 1, 2, 3, 4, 8, 12, 16, 24 ore). 
l'escrezione urinaria nelle 24 ore successive alla somministrazione acuta di 2 g di $\mathrm{Al}(\mathrm{OH})_{3}$. I dati confermano che la riserva funzionale, necessaria per eliminare l'eccesso di $\mathrm{Al}$ dopo carico farmacologico, è ridotta nei nefroni residui già in una condizione di IRC iniziale $(19,20)$. Infatti, l'escrezione urinaria minutata di $\mathrm{Al}$ mostra, in $\mathrm{C}$, un progressivo notevole aumento, già dalla $\mathrm{I}$ e fino alla VIII ora (fino a circa 20 volte $\mathrm{i}$ valori basali); in IRC, invece, tale incremento inizia in ritardo (VIII ora) ed è notevolmente inferiore ( $1.5 \mathrm{vol}$ te $\mathrm{i}$ valori basali). Inoltre la risposta al carico farmacologico acuto è, oltre che di modesta entità in IRC rispetto ai controlli anche ritardata nel tempo. In paticolare, infatti, mentre nei controlli il carico farmacologico di $\mathrm{Al}$ viene eliminato entro le prime 2-8 ore, in IRC necessita di tempi anche superiori alle 24 ore $(21,22)$.

La ritenzione di $\mathrm{P}$ è usuale in IRC. Il $P$ plasmatico, in tal caso, può essere controllato riducendone l'apporto dietetico e diminuendone l'assorbimento intestinale con i chelanti (14). Il chelante più usato in passato è stato sicuramente l'idrossido di alluminio, il cui uso prolungato può causare un'intossicazione da $\mathrm{Al}(23,24)$. Perciò, abbiamo voluto valutare anche le modificazioni che subivano i livelli plasmatici e l'escrezione urinaria di $\mathrm{P}$, dopo la somministrazione acuta di una dose di idrossido di alluminio pari a 2 g. I dati del nostro studio dimostrano che i livelli di P-s, in IRC, si riducono lievemente e senza una significatività statistica, dopo l'assunzione del farmaco, mentre permangono pressoché immodificati in C. Fe-P è, già prima del carico, notevolmente aumentato in IRC rispetto a $\mathrm{C} \mathrm{e}$, dopo il carico, vi è una sua riduzione non significativa in entrambi i gruppi. Infine, l'escrezione urinaria minutata di $\mathrm{P}$ mostra, in $\mathrm{C}$, un aumento di 1.5 volte al tempo di $1 \mathrm{~h}$, significativo $(p<0.05)$, mentre non ha alcuna variazione in IRC.

In conclusione, già nella fase iniziale di IRC si può verificare un accumulo di Al, soprattutto in caso di sovraesposizione. Inoltre, anche la riserva funzionale dei nefroni residui, atta ad eliminare $\mathrm{Al}$ dopo carico farmacologico acuto o cronico $(6-9,14,16-18)$, è ridotta già nell'IRC di grado lievemoderato.

Infine, anche alla luce delle recenti esperienze in trapiantati con $\mathrm{Al}$ overload (25), nella valutazione della risposta immune cellulare in IRC con intossicazione da $\mathrm{Al}(26)$ e nel determinismo della porfiria in uremia (2830) è opportuno riconsiderare l'attenzione che i nefrologi dedicano oggi a tali problematiche, anche nelle fasi iniziali di IRC.

\section{BIBLIOGRAFIA}

1. Meymon H, Cassidy MJD. Gastrointestinal absorption of aluminium. Nephron 1990; 55: 235-6.

2. Ihle BU, Becker GJ. Gastrointestinal absorption of aluminium. Am J Kidney Dis 1985; 6: 302-5.

3. Ott SM. Aluminium accumulation in individual with normal renal function. Am J Kidney Dis 1985; 5: 297-301.

4. Kaehny WD, Higg AP, Jackson JT, et al. Gastrointestinal absorption of aluminium from $\mathrm{Al}$-containing antiacids. N Engl J Med 1977; 296: 1389-90.

5. Partridge NA, Regnier FE, White JL, et al. Influence of dietary constituents on intestinal absorption of aluminium. Kidney Int 1989; 35: 1413-7.

6. Di Iorio B, Terracciano V, Bruno A, et al. Aluminum and phosphorus urinary excretion after modifying gastric acid secretion in normal subjects. Trace Elements in Medicine 1996; 13: 47-9.

7. Di Iorio B, Bruno A, Altieri C, et al. Riduzione dell'escrezione urinaria di alluminio dopo modificazioni dell'acidità gastrica. Giorn It Nefrol 1995; 12: 151-2.

8. Di Iorio B., Bruno A, Terracciano V, et al. Effect of omeprazole on the urinary excretion of $\mathrm{Al}$ and $\mathrm{P}$ in CRF. Nephron 1998; 78: 352-3.

9. Di Iorio B, Terracciano V, Bruno A, et al. Aluminum and phosphorus urinary excretion after modifying gastric acid secretion in CRF. Trace Elements in Medicine 1996; 13: 96-101.

10. Di Iorio B, Bruno A, Terracciano V, et al. Escrezione urinaria di alluminio e fosforo in pazienti con insufficienza renale cronica dopo modificazioni dell' acidità gastrica. Giorn It Nefrol 1996; 13: 115-6. 
11. Canavese C. La tossicità dell'alluminio nei pazienti uremici in trattamento dialitico. Ital J Min Electrol Metab 1990; 4: 201-14.

12. Sàrszegi Z, Jobst K, Nagy J. Gastrointestinal absorption of aluminium in chronic renal failure. $\mathrm{Ab}$ stracts XXXII Congress EDTA, Athens, Greece 11-14 June 1995. Nephrol Dial Transplant 1995; 10: 990.

13. Jobst K, Nagy J. Can Al-containing antacids produce dementia? Nephron 1995; 71: 473.

14. Barsotti G, Morelli E, Guiducci A, et al. Reversal of hyperparathyroidism in severe uremic following very low-protein and low-phosphate diet. Nephron 1982; 30: 310-13.

15. Di Iorio B. Intossicazione da $\mathrm{Al}$ nei pazienti uremici. Federazione Medica 1994; 4: 103-6.

16. Di Iorio B, Gaudiano G, Altieri $\mathrm{C}$, et al. Aluminum urinary excretion in patients with chronic renal failure in treatment with conservative conventional therapy and with ketoanalogs: research on fasting patients and after a pharmacological load. Nephron 1995; 71: 371-2.

17. Di Iorio B, Bruno A, Gaudiano $\mathrm{G}$, et al. Valutazione dell'escrezione di Al nei pazienti con vario grado di IRC e in trattamento con terapia conservativa convenzionale e con chetoanaloghi. Giorn It Nefrol 1994; 19: 427-8.

18. Di Iorio B, Bruno A, Altieri C, et al. Urinary aluminum excretion in patients with CRF in treatment with conservative conventional therapy and with ketoanalogs: hormonal changes? Trace Elements in Medicine 1996; 13: 143-6.

19. Di Iorio B, Terracciano V, Lopez T. Effects of moderate CRF in $\mathrm{Al}$ and $\mathrm{P}$ excretion. Nephron 1998; 79: 111-2.

20. Di Iorio B, Bruno A, Terracciano $\mathrm{V}$, et al. Valutazione cinetica dell'escrezione urinaria di $\mathrm{Al}$ e $\mathrm{P}$ in pazienti con moderata IRC dopo carico acuto di idrossido di Al. Giorn It Nefrol 1997; 14: 117-24.
21. Di Iorio B, Bruno A, Terracciano V, et al. Aluminium excretion in CRF after acute pharmacological load. Ital J Miner Metab 1997; 11: 29-31.

22. Di Iorio B, Bruno A, Terracciano $\mathrm{V}$, et al. Effect of $\mathrm{Al}$ administration in CRF. Trace Elements in Medicine 1997; 14: 72-5.

23. Alfrey CA. Al intoxication. N Engl J Med 1984; 310: 1113-5.

24. De Broe ME, D'Haese PC, Cutteneye MM, et al. New insights and strategies in the diagnosis and treatment of $\mathrm{Al}$ overload in dialysis patients. Nephrol Dial Transplant 1993; 8: S1: 45-50.

25. Garay G, Grosso S, Douthat $\mathrm{W}$, et al. Influence of aluminium overload on the course of post-transplant parathyroid function. Nephrol Dial Transplant 1996; 11 (suppl 3): 65-8.

26. Tzanno-Martins C, Azevedo LS, Orii N, et al. The role of experimental chronic renal failure and aluminium intoxication in cellular immune response. Nephrol Dial Transplant 1996; 11: 474-80.

27. Gafter U, Mamet R, Korzet A, et al. Bollous dermatosis of ESRD: a possible association between abnormal porphyrin metabolism and Al. Nephrol Dial Transplant 1996; 11: 1787-91.

28. Berlyne GM, Yagil R. Aluminum induced porphyria in rats. Lancet 1973; II: 1501-2.

29. Bia MJ, Cooper K, Schall S, et al. Al-induced anemia:pathogenesis and treatment in patients in chronic hemodialysis. Kidney Int 1989; 36: 852-8.

30. Di Iorio B, Terracciano V. Bollous dermatosis in ESRD. Al, iron and erythropoietin. Trace Elements in Medicine 1997; 14: 162. 